\title{
Midgut Epithelial Dynamics Are Central to Mosquitoes' Physiology and Fitness, and to the Transmission of Vector-Borne Disease
}

\author{
Bretta Hixson, Mabel Laline Taracena and Nicolas Buchon* \\ Department of Entomology. Cornell Institute of Host-Microbe Interactions and Disease, College of Agriculture and Life \\ Sciences, Cornell University, Ithaca, NY, United States
}

Keywords: Aedes, Anopheles, mosquito, vector, intestinal stem cell, midgut epithelium, tolerance, resistance

\section{OPEN ACCESS \\ Edited by: \\ Saravanan Thangamani, Upstate Medical University, United States \\ Reviewed by: Maria Luisa Simões, Johns Hopkins University, United States Alexander Franz, \\ University of Missouri, United States \\ *Correspondence: Nicolas Buchon nicolas.buchon@cornell.edu}

Specialty section: This article was submitted to Parasite and Host, a section of the journal Frontiers in Cellular and Infection Microbiology

Received: 13 January 2021 Accepted: 23 February 2021 Published: 25 March 2021

Citation: Hixson B, Taracena ML and Buchon N (2021) Midgut Epithelial Dynamics Are Central to Mosquitoes' Physiology and

Fitness, and to the Transmission of Vector-Borne Disease. Front. Cell. Infect. Microbiol. 11:653156. doi: $10.3389 /$ fcimb.2021.653156

\section{INTRODUCTION}

Hematophagous mosquitoes vector many important human diseases, and a detailed understanding of their physiology is crucial for designing efficient vector-control strategies. The midgut epithelium plays a central role in mosquito physiology: as a digestive tissue, it transitions between processing diets of sugar and blood to support both nutrition and reproduction; as a niche for microbiota, it balances immunity and tolerance to maintain a functional microbiome; and in its capacity as an interface between the mosquito and human pathogens, it serves as a barrier that often bottlenecks parasites as they travel from gut lumen to hemocoel, en route to the salivary glands and their next host (Smith et al., 2014; Franz et al., 2015).

Studies in model organisms, such as Drosophila, have revealed that the insect gut epithelium can be highly complex and dynamic. The Drosophila midgut epithelium comprises diverse cell types, including polyploid enterocytes (ECs), enteroendocrine cells (EEs), and undifferentiated progenitors (intestinal stem cells, ISCs, and enteroblasts, EBs) (Bonfini et al., 2016). These cells communicate and coordinate to maintain the gut's function and integrity. Drosophila ISCs respond to diverse stimuli with symmetric and asymmetric divisions to continuously replenish the midgut epithelium or plastically alter its structure (O’Brien et al., 2011). Inputs including nutrition (O’Brien et al., 2011), endocrine signaling (Reiff et al., 2015; Ahmed et al., 2020), the presence/composition of the microbial community (Buchon et al., 2009a), pathogenic challenge (Buchon et al., 2009b; Houtz et al., 2017), reactive oxygen species (Hochmuth et al., 2011), and aging (Biteau et al., 2008) dynamically alter the kinetics of division, differentiation, and endocycling to reshape epithelial composition.

In contrast to Drosophila, the epithelial composition and dynamics of mosquito midguts are little studied. Early histological studies noted EEs in the mosquito midgut epithelium (Hecker, 1977; Brown et al., 1985), and mapped their production of neuropeptides (Veenstra et al., 1995), but their physiological significance remains unexplored. Likewise, the presence of putative "regenerative cells" in the adult midgut epithelium was noted, but they were believed to be mitotically inactive (Billingsley, 1990). However, a growing number of publications have documented DNA synthesis and/or mitoses in the midguts of several mosquito species (Baton and Ranford-Cartwright, 2007; Hernández-Martínez et al., 2013; Janeh et al., 2017; Serrato-Salas et al., 2018; Taracena et al., 2018; Janeh et al., 2019; Maya-Maldonado et al., 2020), indicating that the mosquito midgut epithelium is 
also highly responsive and dynamic. Furthermore, a recent study using single-cell RNA-sequencing of the midgut of Aedes aegypti revealed multiple cell clusters that express markers corresponding to all major cell types described in the Drosophila midgut (Cui and Franz, 2020). Here, we discuss several mechanisms by which we anticipate that mosquito midgut epithelial dynamics may influence the parameters of fitness, vector competence, and vectorial capacity. We propose that midgut epithelial dynamics are an important and underexplored frontier in the study of mosquito physiology.

\section{EPITHELIAL DYNAMICS IN GUT MATURATION, BLOOD-FEEDING, AGING, AND INTERACTIONS WITH THE GUT MICROBIOTA}

The mosquito midgut begins in the larval stage as a lattice of diploid regenerative cells and larger endoreplicating cells (Ray et al., 2009). During the larval to pupal molt, all polyploid cells are lost, leaving only a network of diploid cells (Nishiura et al., 2003). This pool of cells is likely homologous to the adult midgut progenitors in Drosophila, which give rise to the adult midgut in the final stage of development (Hartenstein et al., 1992; Houtz et al., 2019). In Anopheles albimanus mosquitoes, it was observed that the adult midgut continues to mature in the 24 hours following emergence, as proportions of diploid cells fall, and polyploid cells ( $4 \mathrm{~N}$ and $8 \mathrm{~N}$ ) accumulate via endocycling (MayaMaldonado et al., 2020). This post-emergence maturation phase is marked in mosquitoes by elevated titers of juvenile hormone $(\mathrm{JH})$ (Zhu and Noriega, 2016). Within a few days of emergence, the adult female is ready to take her first blood-meal, stimulating the production of 20 hydroxyecdysone $(20 \mathrm{E})$, which circulates in the hemolymph and activates vitellogenesis in the fat body (Martín et al., 2001; Wang et al., 2002; Bai et al., 2010). In Drosophila, both $\mathrm{JH}$ and $20 \mathrm{E}$ are induced by mating, and both promote epithelial proliferation to drive gut growth (Reiff et al., 2015; Ahmed et al., 2020). Loss of either signal compromises fecundity, suggesting that the gut's growth response is adaptive for optimizing nutrient acquisition to maximize reproductive output. In the mosquito midgut epithelium, there is some evidence that blood-feeding stimulates compositional changes. In the Aedes albopictus midgut, blood-feeding induces phosphorylation of ERK, a kinase in the EGFR pathway (Liu et al., 2020) which, in Drosophila ISCs, is sufficient to drive proliferation (Buchon et al., 2010). We propose that the pro-proliferative response to JH and $20 \mathrm{E}$ may be conserved in the midguts of mosquito species, helping to prepare the epithelium for the task of digesting blood.

Upon acquisition of a blood-meal, the mosquito midgut must pivot to the exploitation of its new diet. A recent single-cell RNAseq study of Ae. aegypti midguts, before and after a blood meal, demonstrated that blood-feeding stimulates an increase in the proportion of putative ISCs/EBs as well as ECs and "EC-like" cells, providing further evidence for a proliferative response (Cui and Franz, 2020). The authors also observed multiple distinct populations of ECs and found that the proportions of EC populations changed in response to blood-feeding. Together, these results suggest that blood-feeding prompts not only the proliferation of progenitors, but changes among differentiated ECs. We propose that these changes could reflect a form of terminal differentiation for immature ECs, which may be key to the digestion of blood.

Midgut epithelial dynamics play an important role in every stage of the insect lifecycle, including its terminus. In Drosophila, the aging of the gut epithelium, with associated dysplasia and loss of barrier integrity, is believed to be an important intrinsic limiting factor in the lifespan of the insect (Biteau et al., 2008; Rera et al., 2012). This aging is accelerated by the presence of the microbiota, which increases the rate of epithelial turnover (Buchon et al., 2009a), and pathogenic microbes can drive even more rapid turnover of the gut epithelium (Buchon et al., 2009b). Considering that the female mosquito midgut is subjected not only to all the ordinary stresses of aging but also to the rigors of blood-feeding - periods of intense mechanical strain accompanied by the rapid proliferation of gut microbes we posit that the midgut may set the limits of mosquito longevity and, by extension, vectorial capacity.

\section{EPITHELIAL DYNAMICS AND CONTROL OF INFECTION}

A mosquito's competence as a vector depends on the successful invasion and traversal of midgut epithelial cells by orally acquired pathogens. It has already been shown that epithelial dynamics, in the form of cell sacrifice, participate in the bottlenecking of invading pathogens in mosquito midguts. In the case of Plasmodium infection, this phenomenon has been likened to a "time bomb" where the invasion of an epithelial cell by an ookinete commences a countdown culminating in the death and extrusion of the invaded cell (Han et al., 2000). Ookinetes that fail to make their way to the safety of the basal lamina before this extrusion occurs are denied advancement to the oocyst stage of development. By sacrificing epithelial cells, the gut may limit or altogether block the progression of plasmodial infection. By a similar principle, the elimination of epithelial cells may also help to limit viral infections in mosquitoes. Several histological studies have associated viral infection of the midgut with epithelial pathology and cell loss (Weaver et al., 1992; Vaidyanathan and Scott, 2006). There is some evidence that this phenomenon is strain-dependent, and correlates negatively with susceptibility to viral infection. Transcriptomic profiling of the midguts of mosquitoes infected with DENV2 found that a refractory strain showed biased representation of transcripts associated with cell death as compared to a susceptible strain (Behura et al., 2011), and a follow-up study found that silencing of pro-apoptotic genes increased the susceptibility of a partially refractory strain (Ocampo et al., 2013). These observations suggest that epithelial cell elimination may underlie the refractoriness exhibited by some strains to infection with incompatible viruses.

Even in the absence of cell sacrifice, we can envision scenarios in which the dynamics of the epithelial response to an incipient infection might alter its outcome. Intensified endocycling might increase immune capacity. Increased proliferation might alter 
midgut epithelial composition and the density of host factors required for epithelial invasion. Newly differentiated ECs might be either more or less resistant to infection than their older counterparts. One 2018 study correlated population-level DENV susceptibility to the timing of the proliferative response in the Ae. aegypti midgut, suggesting that the activity of progenitor cells might influence the outcome of infection (Taracena et al., 2018). We propose that epithelial dynamics may play an important role in the bottlenecking of all kinds of pathogens and helps to limit vector competence in a species and/or strain-dependent manner.

\section{EPITHELIAL REPAIR AND INFECTION TOLERANCE}

While cell sacrifice may help to limit infection, the loss of midgut epithelial cells may negatively impact the survival of the mosquito vector. The time bomb model of Plasmodium invasion dictates that every invaded epithelial cell is fated to die (Han et al., 2000). As the majority of invading ookinetes never mature into oocysts (Ghosh et al., 2000), and as ookinetes may move laterally through multiple ECs during invasion (Han et al., 2000), oocyst counts - ranging from 1-10 in the field (Gouagna et al., 1998) to hundreds in the laboratory (Usui et al., 2011) - represent an extremely conservative estimate of EC loss. As previously noted, infection with viral and bacterial pathogens can also drive EC loss. For the mosquito gut, these losses could destroy a substantial proportion of the epithelium; moreover, even a mild infection and the extrusion of a handful of ECs could theoretically result in a fatal loss of gut barrier integrity.

Given the damage that Plasmodium infection causes to the midgut, it is remarkable that survival effects in Plasmodiuminfected mosquitoes appear to be minimal (Han et al., 2000). Despite a potentially significant loss of midgut epithelium, mosquitoes are apparently able to maintain barrier integrity and gut function sufficient to their needs. Mechanisms have been articulated for closing emerging holes in the gut at the moment of EC loss by drawing together neighboring ECs (Han et al., 2000; Gupta et al., 2005), but it is unlikely that this process fully compensates for cell loss. While the midgut may possess ECs significantly in excess of what it needs to maintain adequate function for survival, it is also possible that mosquitoes' tolerance of midgut damage is dependent on progenitor-mediated replacement of lost epithelial cells. Multiple studies support a homeostatic proliferative role for epithelial progenitors in mosquito vector species. A histological study positively correlated instances of apparent mitosis in the midgut with the intensity of Plasmodium infection in Anopheles stephensi (Baton and Ranford-Cartwright, 2007). Ae. albopictus and Culex pipiens mosquitoes exhibit increased mitoses following oral bacterial infection and chemical challenge (Janeh et al., 2019). Epithelial repair may also be important in response to cell loss sustained during viral infection in non-permissive strains. Even in cases where it does not induce cell loss, viral infection of midgut cells may cause stress and prompt cell signaling responses (e.g. JAK-STAT activation (Behura et al., 2011)) which could stimulate the activity of progenitors. In
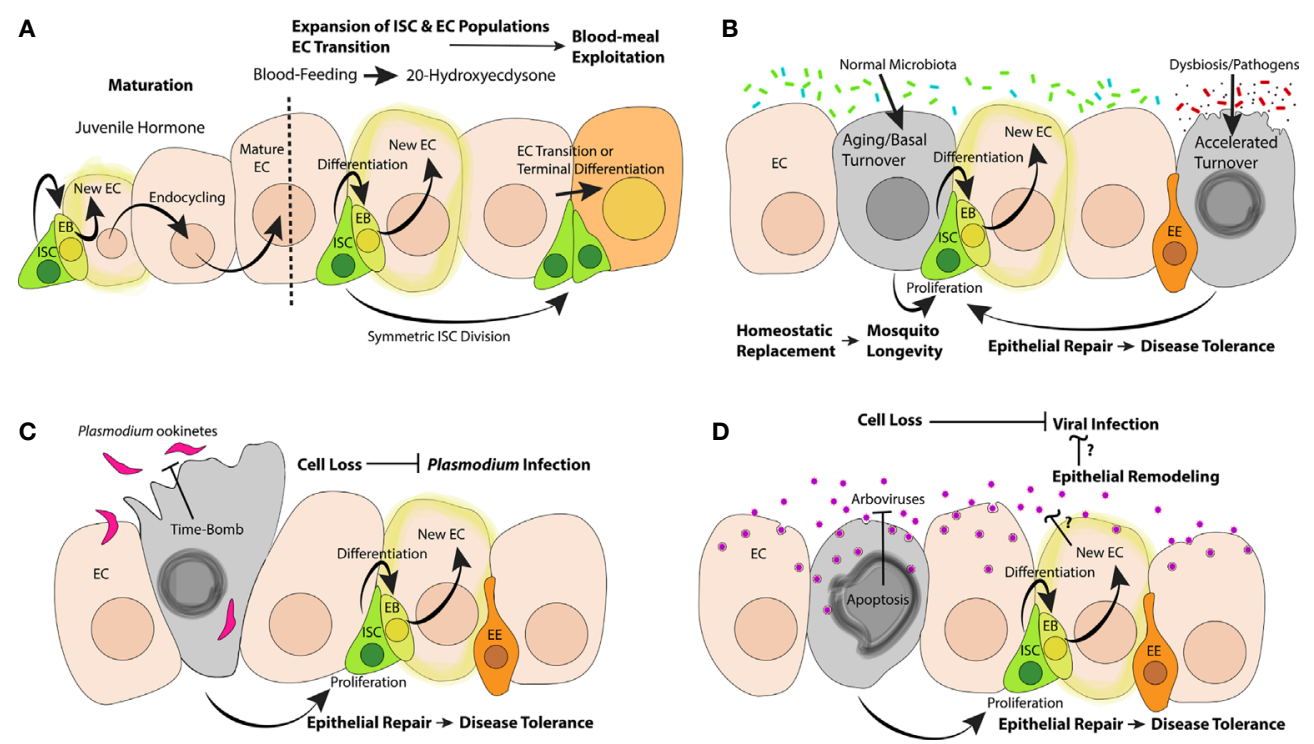

FIGURE 1 | Possible impacts of epithelial dynamics in the mosquito midgut on the hematophagous lifecycle, aging, interactions with gut flora, Plasmodium and arboviral infections. (A) During the post-emergence maturation, JH could stimulate ISCs to proliferate and create new ECs or prompt ECs to endocycle to attain higher ploidy; blood-feeding stimulates the production of 20E, which could stimulate the proliferation of ISCs, the differentiation of new ECs, and transcriptional changes in ECs. (B) Normal microbiota could contribute to aging and basal turnover of EC populations; dying ECs could stimulate ISCs to effect homeostatic replacement; dysbiosis and/or infection with oral bacterial pathogens could accelerate the turnover of epithelial cells; ISC-mediated repair could serve as a disease tolerance mechanism, promoting mosquito survival. Invasion by Plasmodium (C) and/or arboviral pathogens (D) could prompt cell sacrifice mechanisms to limit pathogenic success; ISC proliferation and differentiation could help infected mosquitoes to tolerate epithelial damage. 
summary, we propose that the ability to effect proliferative repair may be important for preserving midgut barrier integrity, with potential knock-on effects for mosquito survival and, hence, vectorial capacity.

\section{DISCUSSION}

The successful transmission of mosquito-borne pathogens depends on (a) the maintenance of a sufficiently large and fit vector population (b) the ability of pathogens to progress from oral to systemic infection (essential for vector competence) and (c) the longevity of infected mosquitoes (a key determinant of vectorial capacity). We propose that midgut epithelial dynamics (cell loss, proliferation, differentiation, and endocycling, Figure 1) may play an important role in determining the rate of pathogen transmission by: (a) adapting epithelial composition according to hormonal and/or nutritional cues to optimize the exploitation of blood meals, thereby maximizing fecundity (b) setting the natural limits of mosquito lifespan (c) suppressing

\section{REFERENCES}

Ahmed, S. M. H., Maldera, J. A., Krunic, D., Paiva-Silva, G. O., Pénalva, C., Teleman, A. A., et al. (2020). Fitness trade-offs incurred by ovary-to-gut steroid signalling in Drosophila. Nature 584, 415-419. doi: 10.1038/s41586-020-2462-y

Bai, H., Gelman, D. B., and Palli, S. R. (2010). Mode of action of methoprene in affecting female reproduction in the African malaria mosquito, Anopheles gambiae. Pest Manage. Sci. 66, 936-943. doi: 10.1002/ps.1962

Baton, L. A., and Ranford-Cartwright, L. C. (2007). Morphological evidence for proliferative regeneration of the Anopheles stephensi midgut epithelium following Plasmodium falciparum ookinete invasion. J. Invertebr. Pathol. 96, 244-254. doi: 10.1016/j.jip.2007.05.005

Behura, S. K., Gomez-Machorro, C., Harker, B. W., deBruyn, B., Lovin, D. D., Hemme, R. R., et al. (2011). Global cross-talk of genes of the mosquito Aedes aegypti in response to dengue virus infection. PloS Negl. Trop. Dis. 5, e1385. doi: 10.1371/journal.pntd.0001385

Billingsley, P. F. (1990). The midgut ultrastructure of hematophagous insects. Annu. Rev. Entomol. 35, 219-248. doi: 10.1146/annurev.en.35.010190.001251

Biteau, B., Hochmuth, C. E., and Jasper, H. (2008). JNK Activity in Somatic Stem Cells Causes Loss of Tissue Homeostasis in the Aging Drosophila Gut. Cell Stem Cell 3, 442-455. doi: 10.1016/j.stem.2008.07.024

Bonfini, A., Liu, X., and Buchon, N. (2016). From pathogens to microbiota: How Drosophila intestinal stem cells react to gut microbes. Dev. Comp. Immunol. 64, 22-38. doi: 10.1016/j.dci.2016.02.008

Brown, M. R., Raikhel, A. S., and Lea, A. O. (1985). Ultrastructure of midgut endocrine cells in the adult mosquito, Aedes aegypti. Tissue Cell 17, 709-721. doi: 10.1016/0040-8166(85)90006-0

Buchon, N., Broderick, N. A., Chakrabarti, S., and Lemaitre, B. (2009a). Invasive and indigenous microbiota impact intestinal stem cell activity through multiple pathways in Drosophila. Genes Dev. 23, 2333-2344. doi: 10.1101/gad.1827009

Buchon, N., Broderick, N. A., Poidevin, M., Pradervand, S., and Lemaitre, B. (2009b). Drosophila intestinal response to bacterial infection: activation of host defense and stem cell proliferation. Cell Host Microbe 5, 200-211. doi: 10.1016/ j.chom.2009.01.003

Buchon, N., Broderick, N. A., Kuraishi, T., and Lemaitre, B. (2010). Drosophila EGFR pathway coordinates stem cell proliferation and gut remodeling following infection. BMC Biol. 8, 152. doi: 10.1186/1741-7007-8-152

Cui, Y., and Franz, A. W. E. (2020). Heterogeneity of midgut cells and their differential responses to blood meal ingestion by the mosquito, Aedes aegypti. Insect Biochem. Mol. Biol. 127, 103496. doi: 10.1016/j.ibmb.2020.103496 and killing pathogens as they traverse the midgut barrier and (d) promoting the survival of infected mosquitoes via repair mechanisms which help the mosquito to tolerate pathogenmediated damage. Understanding these dynamics in mosquitos may allow us to develop interventions that will suppress mosquito fecundity, raise barriers to systemic infection, and/or abbreviate the survival of infected mosquitoes.

\section{AUTHOR CONTRIBUTIONS}

All authors contributed to the article and approved the submitted version.

\section{FUNDING}

\section{NIH R01AI148529, NIH R21AG065733, NIH R01AI148541 and NSF IOS1656118.}

Franz, A. W. E., Kantor, A. M., Passarelli, A. L., and Clem, R. J. (2015). Tissue Barriers to Arbovirus Infection in Mosquitoes. Viruses 7, 3741-3767. doi: 10.3390/v7072795

Ghosh, A., Edwards, M. J., and Jacobs-Lorena, M. (2000). The journey of the malaria parasite in the mosquito: Hopes for the new century. Parasitol. Today 16, 196-201. doi: 10.1016/S0169-4758(99)01626-9

Gouagna, L. C., Gouagna, L. C., Mulder, B., Mulder, B., Noubissi, E., Noubissi, E., et al. (1998). The early sporogonic cycle of Plasmodium falciparum in laboratory-infected Anopheles gambiae: an estimation of parasite efficacy. Trop. Med. Int. Heal. 3, 21-28. doi: 10.1046/j.1365-3156.1998.00156.x

Gupta, L., Kumar, S., Han, Y. S., Pimenta, P. F. P., and Barillas-Mury, C. (2005). Midgut epithelial responses of different mosquito-Plasmodium combinations: the actin cone zipper repair mechanism in Aedes aegypti. Proc. Natl. Acad. Sci. U. S. A. 102, 4010-4015. doi: 10.1073/pnas.0409642102

Han, Y. S., Thompson, J., Kafatos, F. C., and Barillas-Mury, C. (2000). Molecular interactions between Anopheles stephensi midgut cells and Plasmodium berghei: the time bomb theory of ookinete invasion of mosquitoes. EMBO J. 19, 6030-6040. doi: 10.1093/emboj/19.22.6030

Hartenstein, A. Y., Rugendorff, A., Tepass, U., and Hartenstein, V. (1992). The function of the neurogenic genes during epithelial development in the Drosophila embryo. Development 116, 1203-1220.

Hecker, H. (1977). Structure and function of midgut epithelial cells in culicidae mosquitoes (insecta, diptera). Cell Tissue Res. 184, 321-341. doi: 10.1007/ BF00219894

Hernández-Martínez, S., Barradas-Bautista, D., and Rodríguez, M. H. (2013). Diferential Dna Synthesis In Anopheles Albimanus Tissues Induced By Immune Challenge With Different Microorganisms. Arch. Insect Biochem. Physiol. 84, 1-14. doi: 10.1002/arch.21108

Hochmuth, C. E., Biteau, B., Bohmann, D., and Jasper, H. (2011). Redox regulation by Keap1 and Nrf2 controls intestinal stem cell proliferation in Drosophila. Cell Stem Cell 8, 188-199. doi: 10.1016/j.stem.2010.12.006

Houtz, P., Bonfini, A., Liu, X., Revah, J., Guillou, A., Poidevin, M., et al. (2017). Hippo, TGF- $\beta$, and Src-MAPK pathways regulate transcription of the upd3 cytokine in Drosophila enterocytes upon bacterial infection. PloS Genet. 13, e1007091. doi: 10.1371/journal.pgen.1007091

Houtz, P., Bonfini, A., Bing, X., and Buchon, N. (2019). Recruitment of Adult Precursor Cells Underlies Limited Repair of the Infected Larval Midgut in Drosophila. Cell Host Microbe 26, 412-425.e5. doi: 10.1016/j.chom.2019.08.006

Janeh, M., Osman, D., and Kambris, Z. (2017). Damage-induced cell regeneration in the midgut of aedes albopictus mosquitoes. Sci. Rep. 7, 1-10. doi: 10.1038/ srep44594 
Janeh, M., Osman, D., and Kambris, Z. (2019). Comparative Analysis of Midgut Regeneration Capacity and Resistance to Oral Infection in Three DiseaseVector Mosquitoes. Sci. Rep. 9. doi: 10.1038/s41598-019-50994-4

Liu, W.-Q., Chen, S.-Q., Bai, H.-Q., Wei, Q.-M., Zhang, S.-N., Chen, C., et al. (2020). The Ras/ERK signaling pathway couples antimicrobial peptides to mediate resistance to dengue virus in Aedes mosquitoes. PloS Negl. Trop. Dis. 14, e0008660. doi: 10.1371/journal.pntd.0008660

Martín, D., Wang, S. F., and Raikhel, A. S. (2001). The vitellogenin gene of the mosquito Aedes aegypti is a direct target of ecdysteroid receptor. Mol. Cell. Endocrinol. 173, 75-86. doi: 10.1016/S0303-7207(00)00413-5

Maya-Maldonado, K., Cardoso-Jaime, V., Hernández-Martínez, S., VázquezCalzada, C., Hernández-Hernández, F. de la C., and Lanz-Mendoza, H. (2020). DNA synthesis increases during the first hours post-emergence in Anopheles albimanus mosquito midgut. Dev. Comp. Immunol. 112, 103753. doi: $10.1016 /$ j.dci.2020.103753

Nishiura, J. T., Ho, P., and Ray, K. (2003). Methoprene Interferes with Mosquito Midgut Remodeling During Metamorphosis. J. Med. Entomol. 40, 498-507. doi: 10.1603/0022-2585-40.4.498

Ocampo, C. B., Caicedo, P. A., Jaramillo, G., Ursic Bedoya, R., Baron, O., Serrato, I. M., et al. (2013). Differential Expression of Apoptosis Related Genes in Selected Strains of Aedes aegypti with Different Susceptibilities to Dengue Virus. PloS One 8 (4), e61187. doi: 10.1371/journal.pone.0061187

O'Brien, L. E., Soliman, S. S., Li, X., and Bilder, D. (2011). Altered modes of stem cell division drive adaptive intestinal growth. Cell 147, 603-614. doi: 10.1016/ j.cell.2011.08.048

Ray, K., Mercedes, M., Chan, D., Choi, C. Y., and Nishiura, J. T. (2009). Growth and Differentiation of the Larval Mosquito Midgut. J. Insect Sci. 9, 1-13. doi: 10.1673/031.009.5501

Reiff, T., Jacobson, J., Cognigni, P., Antonello, Z., Ballesta, E., Tan, K. J., et al. (2015). Endocrine remodelling of the adult intestine sustains reproduction in drosophila. Elife 4. doi: 10.7554/eLife.06930

Rera, M., Clark, R. I., and Walker, D. W. (2012). Intestinal barrier dysfunction links metabolic and inflammatory markers of aging to death in Drosophila. Proc. Natl. Acad. Sci. U. S. A. 109, 21528-21533. doi: 10.1073/pnas.1215849110

Serrato-Salas, J., Hernández-Martínez, S., Martínez-Barnetche, J., Condé, R., Alvarado-Delgado, A., Zumaya-Estrada, F., et al. (2018). De Novo DNA synthesis in Aedes aegypti midgut cells as a complementary strategy to limit dengue viral replication. Front. Microbiol. 9, 801. doi: 10.3389/fmicb. 2018.00801
Smith, R. C., Vega-Rodríguez, J., and Jacobs-Lorena, M. (2014). The Plasmodium bottleneck: malaria parasite losses in the mosquito vector. Mem. Inst. Oswaldo Cruz 109, 644-661. doi: 10.1590/0074-0276130597

Taracena, M. L., Bottino-Rojas, V., Talyuli, O. A. C., Walter-Nuno, A. B., Oliveira, J. H. M., Angleró-Rodriguez, Y. I., et al. (2018). Regulation of midgut cell proliferation impacts Aedes aegypti susceptibility to dengue virus. PloS Negl. Trop. Dis. 12 (5), e0006498. doi: 10.1371/journal.pntd.0006498

Usui, M., Fukumoto, S., Inoue, N., and Kawazu, S. (2011). Improvement of the observational method for Plasmodium berghei oocysts in the midgut of mosquitoes. Parasitol. Vectors 4, 118. doi: 10.1186/1756-3305-4-118

Vaidyanathan, R., and Scott, T. W. (2006). Apoptosis in mosquito midgut epithelia associated with West Nile virus infection. Apoptosis 11, 1643-1651. doi: 10.1007/s10495-006-8783-y

Veenstra, J. A., Lau, G. W., Agricola, H. J., and Petzel, D. H. (1995). Immunohistological localization of regulatory peptides in the midgut of the female mosquito Aedes aegypti. Histochem. Cell Biol. 104, 337-347. doi: 10.1007/BF01458127

Wang, S. F., Li, C., Sun, G., Zhu, J., and Raikhel, A. S. (2002). Differential expression and regulation by 20-hydroxyecdysone of mosquito ecdysteroid receptor isoforms A and B. Mol. Cell. Endocrinol. 196, 29-42. doi: 10.1016/ S0303-7207(02)00225-3

Weaver, S. C., Lorenz, L. H., and Scott, T. W. (1992). Pathologic changes in the midgut of Culex tarsalis following infection with western equine encephalomyelitis virus. Am. J. Trop. Med. Hyg. 47, 691-701. doi: 10.4269/ ajtmh.1992.47.691

Zhu, J., and Noriega, F. G. (2016). The Role of Juvenile Hormone in Mosquito Development and Reproduction. Adv. Insect Physiol. (Academic Press Inc.) 51, 93-113. doi: 10.1016/bs.aiip.2016.04.005

Conflict of Interest: The authors declare that the research was conducted in the absence of any commercial or financial relationships that could be construed as a potential conflict of interest.

Copyright (c) 2021 Hixson, Taracena and Buchon. This is an open-access article distributed under the terms of the Creative Commons Attribution License (CC BY). The use, distribution or reproduction in other forums is permitted, provided the original author(s) and the copyright owner(s) are credited and that the original publication in this journal is cited, in accordance with accepted academic practice. No use, distribution or reproduction is permitted which does not comply with these terms. 